\title{
Face and Hand Gesture Recognition System for Controlling VLC Media Player
}

\section{K M Bilvika"1, Sneha B K², Sahana K M², Tejaswini S M Patil ${ }^{2}$}

${ }^{1}$ Assistant Professor, Department of Information Science and Engineering, New Horizon College of Engineering, Bangalore, Karnataka, India

2Department of Information Science and Engineering, Visveswaraiah Technological University, New Horizon College of Engineering, Bangalore, Karnataka, India

\section{ABSTRACT}

Article Info

Volume 7, Issue 4

Page Number: 119-124

Publication Issue :

July-August-2021

\section{Article History}

Accepted : 02 July 2021

Published : 08 July 2021
In human-computer interaction or sign language interpretation, recognizing hand gestures and face detection become predominant in computer vision research. The primary goal of this proposed system is to create a system, which can identify hand gestures and facial detection to convey information for controlling media player. For those who are deaf and dumb sign language is a common, efficient and alternative way for talking, by using the hand and facial gestures we can easily understand them. Here hand and face are directly use as the input to the device for effective communication purpose of gesture identification there is no need of an intermediate medium.

Keywords : Webcam, Face detection, Hand gesture Recognition, Pyautogui keyboard controller, VLC media player

\section{INTRODUCTION}

Gesture recognition affords real-time information to a computer to make it fulfill the user's commands. It is an area of active current research in computer vision and machine learning. Generally, interaction between a computer and human is performed with a keyboard and mouse. However, to control and interact with a media player we are using a keyboard and mouse. To make this interaction easier and more convenient without using extra devices such as keyboard and mouse, we propose to build gesturebased interaction for controlling the media player. resources that assist you in writing a professional technical paper.
In this gesture based controlling media player system we are going to recognize both facial and hand gestures. Now a days Media player has become one of the fundamental parts of our daily lives and it is used by anyone and everyone. Generally, media player has functions like volume up, volume down, play, pause, forward, backward and mute operations. This gesture-based interaction controls those functions of a media player through webcam. We will be achieving this through Image Processing and Pyautogui. The image processing by using OpenCV and for further tasks like Gaussian and Morphological transformation. The Pyautogui is a python library that allows to control the mouse and keyboard. We are using Face detection to play and pause the video and Hand gesture recognition for other functions. 


\section{A. Scope of Project}

This is the enhanced media player in which it controls all the operations using face and hand-based gesture recognition. The detection and extraction of the face and hand characteristics during the image stream acquisition, which is obtained from an integrated webcam. This face and gesture-based media player is efficient and it can assist in minimizing human efforts.

- This automated system is to get better experience of using media player.

- This is efficient system in which any part of the video is not missing.

- It provides a quicker way of operation by capturing the images using integrated webcam and automating it.

- This project is helpful to automate the system by controlling operations of media player by face detection and hand recognition through real time capturing image features thorough webcam.

\section{B. Problem Definition}

"To build a system to control Media player through Face detection and Hand Gestures Recognition to make it more efficient and convenient by using OpenCV and Image Processing”.

\section{Objective of the study}

Media player has become one of the fundamental parts of our daily lives. There are lot of critical situations in controlling media player where some people don't have experience of using keyboard and mouse in computer to control media player. We have come up with a small part of such problem to make it easier and convenient to everyone who are not able to use keyboard and mouse in computer using image processing technology and appropriate library commands to control keyboard.

The main objective of the system is to design and develop a Face and Hand Gesture Recognition System on Controlling Media player by making use of OpenCV and Pyautogui library. More specifically, this project works to achieve the following:

- To design a face and hand gesture-based media player system that will provide efficient services such as pause the video when user not looking at the video and plays when user looks at it.

- To reduce the usage of keyboard and mouse in computer.

- To create a media player that is convenient and easily manageable.

- The gesture recognition on controlling media player should be user friendly and provide efficiency.

\section{Proposed System}

The face and hand gesture recognition system on controlling media player works using real time gestures input from user using integrated webcam and provided gesture matches with a function to control the media player.

Working of the project is as follows:

1. First webcam will open and user provides gesture input through that.

2. The VLC app and video which as to be played should be open through subprocess shell command.

3. The face detection should be done through haarcascade_frontalface classifier.

4. If the face detects then the video starts playing continuously, otherwise the video will be pause.

5. Detects and recognize the hand gestures through image processing technique and matches with the keyboard hotkeys provided by Pyautogui library.

6. Perform those operations. 


\section{METHODS AND MATERIAL}

To implement this project, we used integrated webcam to get the input by user. On passing input sequence of images through image processing technique to process the image and obtain the information from images. The model provides basic types of VLC media player functions, that is, play, pause, volume up, volume down, mute, forward and backward the video. This is performed by face and hand gesture recognition. Face detection is to play and pause the video. Hand gesture recognition is to perform other functions.

We make use of different libraries and modules of Python, like OpenCV, NumPy, Pyautogui, math and subprocess to build the application.

- Subprocess module is a library of python used to run new application or program. Here we used subprocess.Popen() function to open VLC application and VLC video using appropriate path of the application and video.

\section{A. System Architecture}

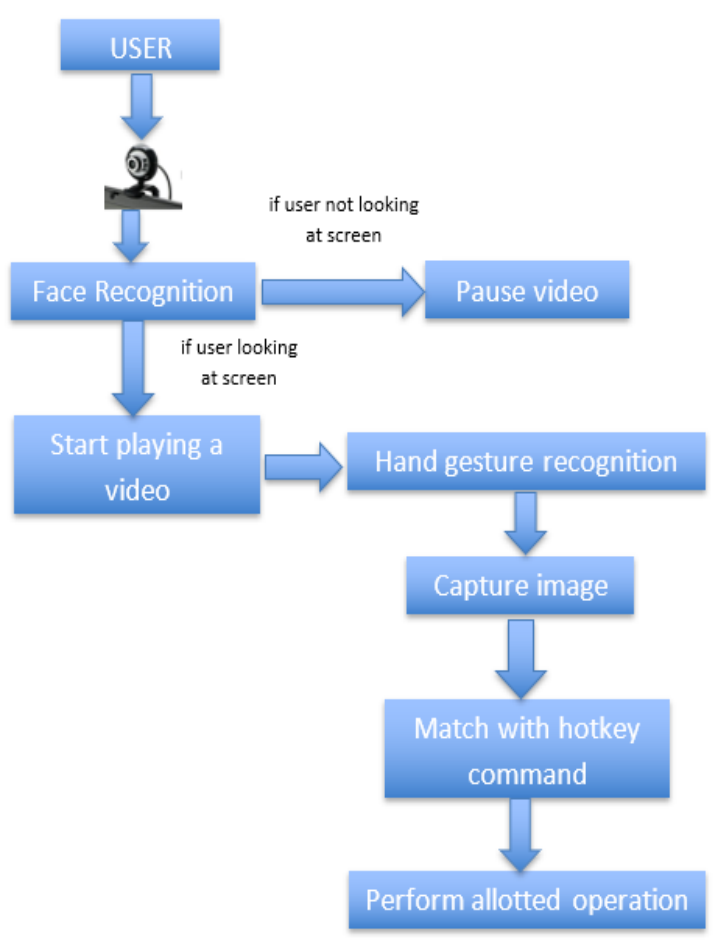

\section{B. Requirements}

1) Hardware Requirements

$\begin{array}{lll}\text { Processor } & - & \text { Intel Core i5 and above } \\ \text { Speed } & - & 2.5 \mathrm{GHz} \\ \text { RAM } & - & 8 \mathrm{~GB}(\mathrm{~min}) \\ \text { Hard Disk } & - & 50 \mathrm{~GB} \\ \text { Webcam } & \\ \text { OS version } 5.0 \text { and above }\end{array}$

2) Software Requirements

$\begin{array}{lll}\text { Operating System } & - & \text { Windows } 10 \\ \text { Programming Language } & - & \text { Python } 3.6 \\ \text { Compiler } & - & \text { Python Idle }\end{array}$

\section{Modules of the project}

1) Face Detection: This module is used to play and pause the video. If webcam detects user's face, then the video will play without any interruption. If the system could not detect user's face, then the video will be stopped immediately.

For this face detection, we are using Haar featurebased cascade classifiers to detect the user's face. It is a machine learning based approach in which a cascade function is trained from a lot of positive and negative images to train the classifier. OpenCV already contains many pre-trained classifiers stored as XML files. For face detection we are using haar cascade classifier that is, "haarcascade_frontalface_default.xml" file, which is used to detect the face.

2) Hand Gesture Recognition: This module is used to perform other functions such as volume up, volume down, mute, forward and backward. Image processing technique is used for Hand gesture recognition. Image processing is the process of analyzing and manipulating an image and it aimed to improving its quality and for extracting information from image. 
Image processing algorithm which is used in this project is,

- Morphological image processing

It removes the imperfections from the binary images because binary regions of binary images produced by simple thresholding can be distorted by noise. It also helps in smoothing the image.

There are two operations of morphological image processing,

- Dilation: This operation adds the pixels to the object boundaries in an image.

- Erosion: This operation removes the pixels from boundaries of the object.

Steps involved in Morphological transformation are:

- First, we need to change colour space from BGR to HSV.

- Create binary image using HSV image.

- Create Kernel for morphological transformation.

- Apply morphological transformation that is erosion and dilation to filter the background noise.

3) Counting Fingers: After detecting hand, we are going to count fingers to perform the appropriate Operations according to number of fingers. To count fingers, we are using contours, convex hull, convexity defects.

Steps involved in counting fingers are:

- First, we need find contours, Contours are boundary of the fingers.

- Draw convex hull for contours, convex hull is the farthest boundary points of a contour.

- Find convexity defects using contours and hull, convexity defect is deviation of the contour from its convex hull.

- Find angle between start and end tip of finger for all defects using cosine rule.

- Count defects to print number of fingers.

- For zero defects, print 1 finger. 1 defect for 2 fingers, 2 defects for 3 fingers, 3 defects for 4 fingers and 4 defects for 5 fingers.
4) Keyboard Controller: To control the VLC media player operations, we are using Pyautogui python library. This library provides programmatical support to control both mouse and keyboard. Keyboard hotkeys, which are able to control operations of VLC media player are passed to the Pyautogui function in the program to control media player without using external devices such as keyboard and mouse.

The keys used are

- 'shift', 'space': To play the video

- 'ctrl', 'space': To pause

- 'volumeup': To increase the volume

- 'volumedown': To decrease the volume

- 'mute': To mute the volume

- 'Ctrl', 'left': To rewind the video

- 'Ctrl', 'right': To forward the video

\section{System Data Flow Diagram}

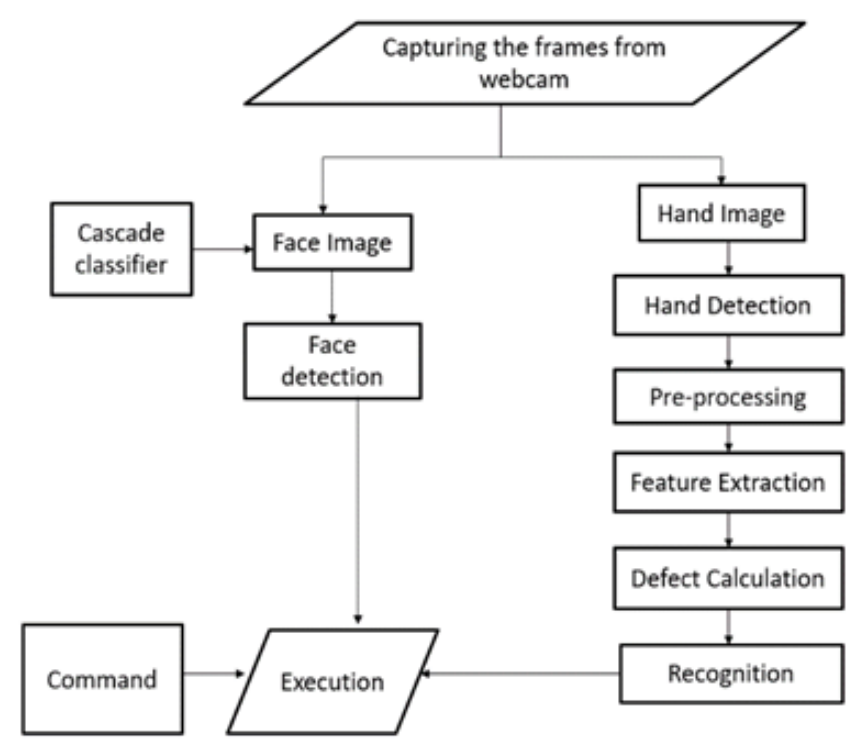




\section{RESULTS AND DISCUSSION}

1. The video is start playing, when face is detected

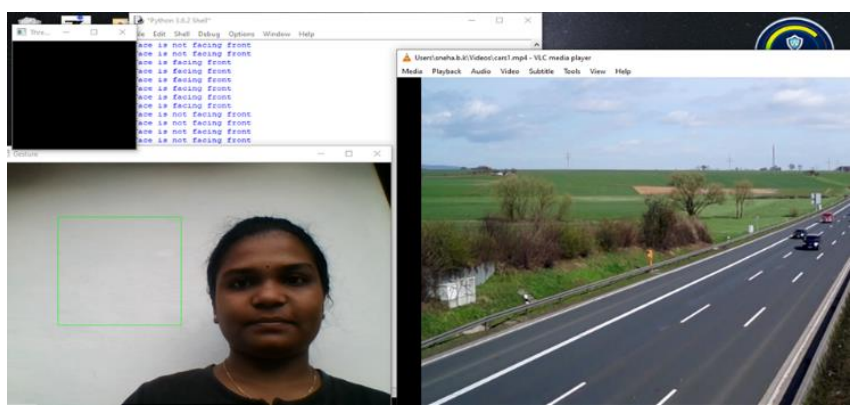

2. The video is paused when face is not detected

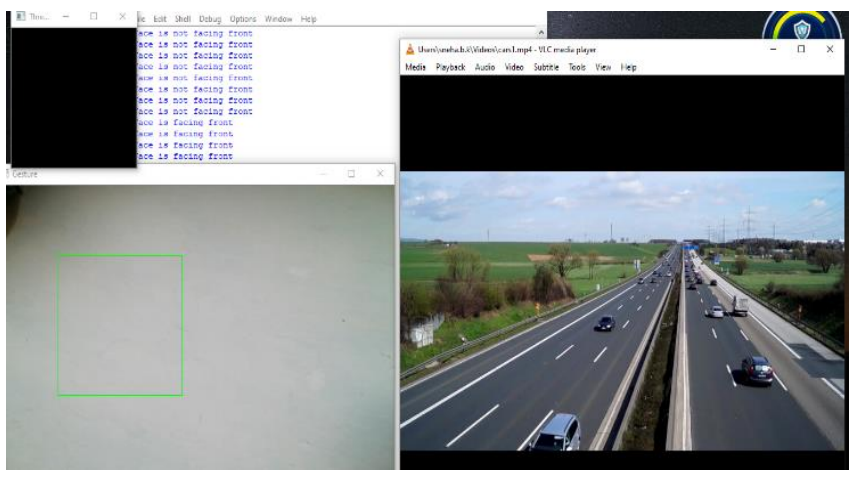

3. When user shows the gesture as one finger, then it increases the volume.

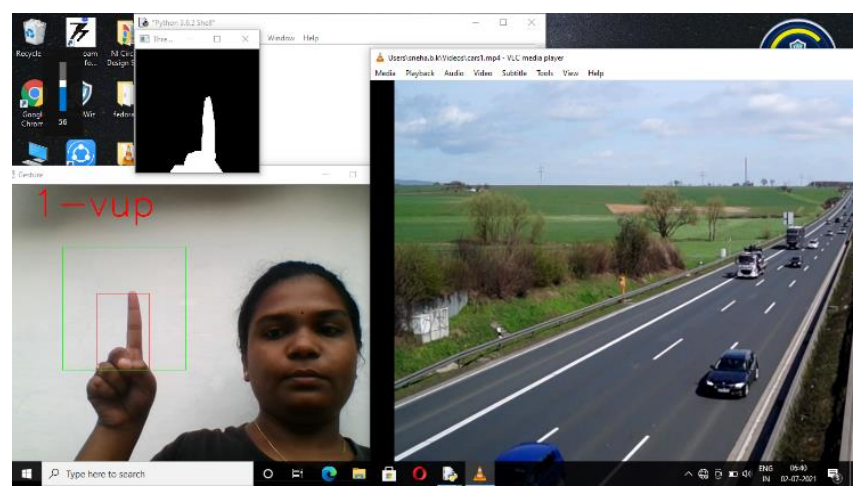

4. When user shows the gesture as one finger, then it increases the volume.

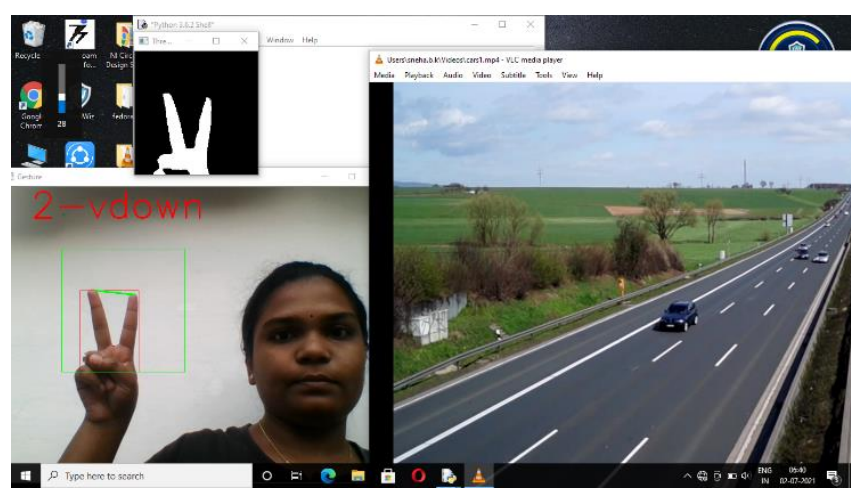

5. When user shows the gesture as one finger, then it increases the volume.

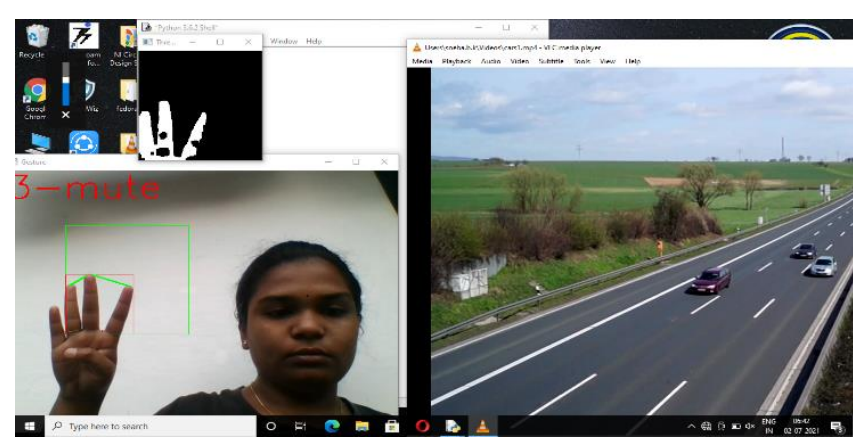

6. When user shows the gesture as one finger, then it increases the volume.

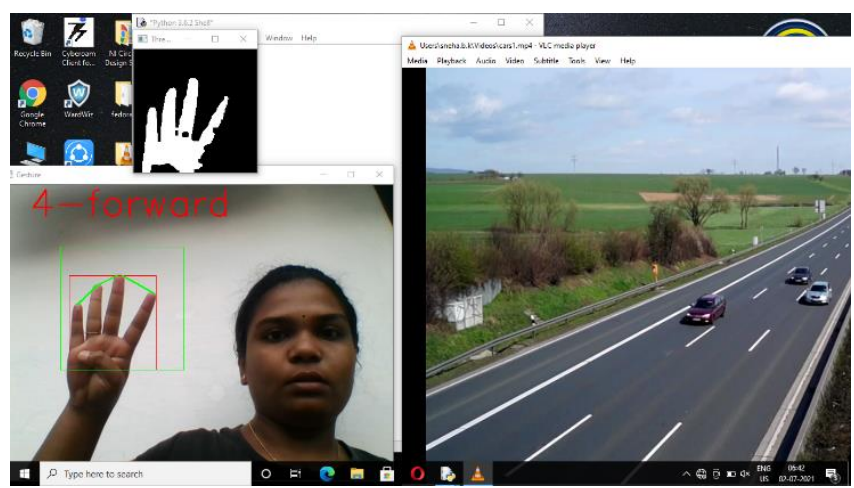


7. When user shows the gesture as one finger, then it increases the volume.

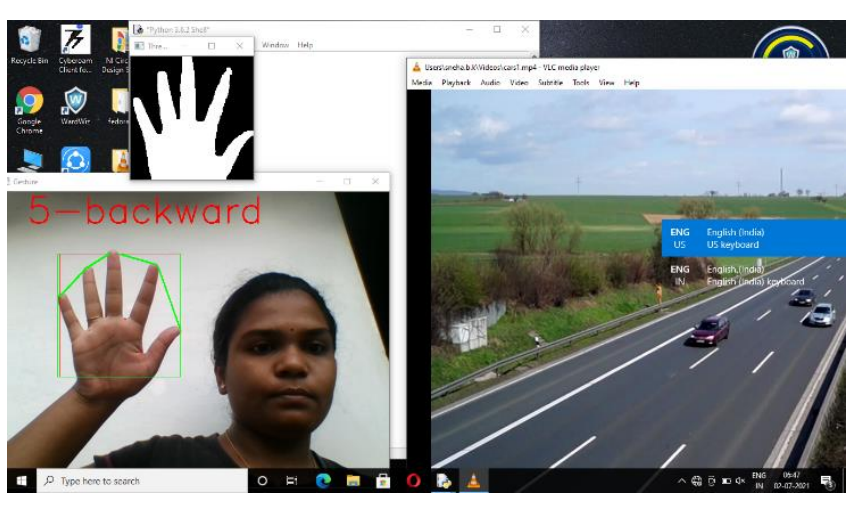

IV. CONCLUSION

The proposed system is to control VLC media player through hand gesture recognition and face detection. This project is more efficient when comparing to the existing VLC media player by making it automated. A better way of controlling media player is implemented. Instead of using keyboard and mouse to control a media player, user controls media player by recognizing the gestures and detecting face, which reduced the use of external devices. This also helped the user to save the cost of buying external devices and saves time. The video starts playing, when it detects a user face. If user gets interrupted, when he/she watching video and face is not detected, it pauses immediately. Hand gesture recognition is to control other operations of video player. This also ensured the user wouldn't lose any part of the video.

\section{REFERENCES}

[1]. Vijay Shinde, Tushar Bacchav, Jitendra Pawar and Mangesh Sanap. 2014. International Journal of Engineering Research \& Technology (IJERT). ISSN NO: 2278-0181 Vol. 3 Issue 1, January-2014

[2]. Rohini M, Abishek Leo Kingston. j, Shriram G S, Siva Sankaran \& Vasuki G, "Hand Gesture Recognition Using OpenCV", International Journal of Scientific Research in Science and
Technology (IJSRST). ISSN NO:2395-602X, DOI:10.32628, March-April 2021.

[3]. B. Garcia, S.A. Viesca, Real-time American sign language recognition with convolutional neural networks, Convolutional Neural Networks for Visual Recognition 2.

[4]. ABBES Zeineb and CHIBANI Chaala "Hand Gesture Recognition System", International Journal of Computer Science and Information Security (IJCSIS). Vol. 14, No. 6, June 2016.

\section{Cite this article as :}

K M Bilvika, Sneha B K, Sahana K M, Tejaswini S M Patil, "Face and Hand Gesture Recognition System for Controlling VLC Media Player", International Journal of Scientific Research in Computer Science, Engineering and Information Technology (IJSRCSEIT), ISSN : 2456-3307, Volume 7 Issue 4, pp. 119-124, July-August 2021. Available at doi : https://doi.org/10.32628/CSEIT217423 Journal URL : https://ijsrcseit.com/CSEIT217423 